\title{
POWER LAW INFLATION WITH A VARYING CONSTANT OF GRAVITATION AND BRANS-DICKE COSMOLOGY
}

\author{
Abu Baker Mohammad Farhad and Abul Kalam Azad \\ Department of Mathematics \\ University of Chittagong, Chittagong, Bangladesh \\ Email: farhadabm@gmail.com
}

Received 13.01.2009

Accepted 22.10.2011

\begin{abstract}
The homogeneous and isotropic Brans-Dicke cosmological solutions satisfying Whitrow Randall's[18] relation which have been discussed recently by Berman and Som[2] are re-examined. We extend their results and present the most general solution by solving a differential equation completely. We show that Mach's assumption leads to power-law solutions in the Euclidean case.
\end{abstract}

\section{Introduction}

In 1961 C. Brans and R.H. Dicke[3,15] provided an interesting alternative to general relativity that was based on Mach's[7] principle. To understand the reasons leading to their field equations, we first note the concept of a variable inertial mass. Masses are measured in certain units, such as masses of elementary particles, which are thousands subject to change! We need an independent unit of mass against which an increase or decrease in mass of a particle can be measured. Such a unit is provided by gravity, by the so called Plank mass encountered as follows [8]:

$$
\left(\frac{\hbar c}{G}\right) \cong 2.16 \times 10^{-5} g
$$

Thus the dimensionless quantity

$\chi=m\left(\frac{G}{\hbar c}\right)^{1 / 2} ;$ measured at different points in space-time can tell us whether masses $m$ are changing. Or alternately, if we insist on using mass units that are the same everywhere, a change of $\chi$ would tell us that the gravitational constant $G$ is changing. This is the conclusion Brans-Dicke drew from their approach to Mach's principle. They looked for a framework in which the gravitional constant $G$ arises from the structure of the universe, so that a changing $G$ could be looked upon as the Machian consequence of a changing universe.

In 1953 D. W. Sciama[5] had given general arguments leading to a relationship between $G$ and the large scale structure of the universe. We have already come across one example of such a relation in the Friedman cosmologies: 
$\rho_{0}=\frac{3 H_{0}^{2}}{4 \pi G} q_{0}$, where $q_{0}$ is the present deceleration parameter. If we write $R_{0}=\frac{C}{H_{0}}$ as a characteristic length of the universe and $M_{0}=4 \pi \rho_{0} \frac{R_{0}^{3}}{3}$ as the characteristic mass of the universe, then the above relation becomes:

$$
\frac{1}{G}=\frac{M_{0}}{R_{0} c^{2}} q_{0}^{-1} \sim \frac{M}{R_{0} c^{2}} \sim \sum \frac{m}{r c^{2}}
$$

Given a dynamical coupling between the inertia and gravity, a relation of the above type is expected to hold. Brans and Dicke[14] took this relation as one that determine $G^{-1}$ from a linear superposition of inertial contributions $m / r^{2} c^{2}$, the typical one being from a mass $m$ at a distance $r$ from the point where $G$ is measured. Since ${ }_{m / r}$ is a solution of a scalar wave equation with a point source of strength $m$, Brans and Dicke[16] postulated that $G$ behaves as the reciprocal of a scalar field $\varphi: G \sim \varphi^{-1}$, where $\varphi$ is expected to satisfy a scalar wave equation whose source is all the matter in the universe.

\section{Models with a Varying Constant of Gravitation}

Gravitational forces are remarkably weak by the standards of atomic or nuclear physics.

For instance, the ratio of the gravitational to the electric force between the electron and the proton has the value [17]

$$
G m_{p} \frac{m_{e}}{e^{2}} \approx 4.4 \times 10^{-40}
$$

The relation among $G, H_{0}$ (Hubble Constant), $m_{p}$ and the present cosmic baryon number density $n_{0}$ :

$$
G n_{0} m_{p} \approx H_{0}^{2}
$$

This is a relation between two cosmological parameters, $n_{0}$ and $H_{0}$, and is the factrequired by various cosmological models, such as the Friedmann models. The Hubble's constant $H_{0}$ is a constant, but a function of the age of the universe. One way of dealing with this problem is to replace $H_{0}$ with a quantity of comparable magnitude that is a constant, for instance, in a closed Friedmann model we can use the reciprocal of time it takes for the universe to expand to its maximum extent, while in a steady state model, the Hubble constant itself will do.

In 1937 a very different approach was suggested by Dirac[13,10]. Dirac chose $G$ as the "constant" 
That varies with time, and in order to preserve $\left(\frac{\hbar^{2} H_{0}}{G c}\right) \approx m_{\pi}$ he proposed that

$$
G \propto \frac{\dot{R}}{R}
$$

In addition, Dirac suggested that relation like (A) also remain true, with a constant factor of proportionality, as the universe expands. Since $n \propto R^{-3}$, i.e., $n \propto \frac{1}{R^{3}}$, it follows that, using the relation (1)

$$
G n_{0} m_{p} \approx H_{0}^{2}
$$

Gives

$$
G R^{-3} \propto \frac{\dot{R}^{2}}{R^{2}}
$$

Eliminating $_{G(t)}$ from (1) and (2) let us proceed as follows:

Putting $_{G}=\frac{\dot{R}}{R}$ in (2) we get

$$
R \propto t^{1 / 3}
$$

Now (1) gives

$$
G \propto t^{-1}
$$

From (3) we have

$$
\begin{aligned}
& R \propto t^{1 / 3} \\
& \text { or, } t_{0}=\frac{1}{3} H_{0}^{-1}
\end{aligned}
$$

Finally, (4) gives a present rate of decrease of the "constant" of gravitation.

$$
\frac{\dot{G}}{G}=-3 H_{0}
$$

The gravitational constant $G$ ought to be related to the average value of a scalar field ${ }_{\phi}$, which is coupled to the mass density of the universe.

The simplest generally covariant field equation for such a scalar field would be

$$
\square \square^{2} \phi=4 \pi \lambda T_{M \mu}^{\mu}
$$

Where $\square \square^{2} \phi=\phi ;^{\rho} ; \rho$ is now the invariant d' Alembertian, $\lambda$ is a coupling constant, and $T_{M}^{\mu \nu}$ is the energy momentum tensor of the matter (i.e., everything but gravitation and the ${ }_{\phi}$-field) of the universe. We can make a rough estimate of the average value of ${ }_{\phi}$ by computing the central potential of a gas sphere with the cosmic mass 
density $\rho \sim 10^{-29} \mathrm{gcm}^{-3}$ and radius equal to the apparent radius of the universe $_{R} \sim 10{ }^{28} \mathrm{~cm}$. This gives an average value

$$
\langle\phi\rangle \sim \lambda \rho R^{2} \sim \lambda \times 10^{27} \mathrm{gcm}^{-1}
$$

Note that $10^{27} \mathrm{gcm}^{-1}$ is reasonably close to the constant $1 / G=1.35 \times 10^{28} \mathrm{gcm}^{-1}$; hence we normalize $\varphi$ so that

$$
\langle\phi\rangle \cong 1 / G
$$

And (8) then shows that $\lambda$ is a dimensionless number of order unity. These considerations led Brans and Dicke to suggest that the correct field equations for gravitation are obtained by replacing $G$ with ${ }_{1 / \phi}$ and including an energy momentum tensor $T_{\phi}^{\mu \nu}$ for the ${ }_{\phi}$-field in the source of the gravitational field:

$$
R^{\mu \nu}-\frac{1}{2} g^{\mu \nu} R=-\frac{8 \pi}{\phi}\left[T_{M}^{\mu \nu}+T_{\phi}^{\mu \nu}\right]
$$

The most general symmetric tensor that can be built up from terms each of which involves two derivatives of one or two ${ }_{\phi}$-fields, and ${ }_{\phi}$ itself, is

$$
T_{\phi}^{\mu}{ }_{v}=A(\phi) \phi ;^{\mu} \phi ;_{v}+B(\phi) \delta_{v}^{\mu} \phi_{; \rho} \phi^{; \rho}+C(\phi) \phi ;^{\mu} ;_{v}+\delta_{v}^{\mu} D(\phi) \square \square^{2} \phi
$$

The unique solution is

$$
\begin{aligned}
A(\phi) & =\frac{\omega}{8 \pi \phi} & B(\phi) & =-\frac{\omega}{16 \pi \phi} \\
C(\phi) & =\frac{1}{8 \pi} & D(\phi) & =-\frac{1}{8 \pi}
\end{aligned}
$$

Where $\omega$ is a convenient dimensionless constant given by

$$
\omega=\frac{1}{\lambda}-\frac{2}{3}
$$

The field equation (7) of the Brans-Dicke[8] theory now read

$$
\square \square^{2} \phi=\frac{8 \pi}{3+2 \omega} T_{M \mu}^{\mu}
$$

is assumed to obey a field equation

$$
\square \square^{2} \phi \equiv\left(\phi ;{ }^{\mu}\right){ }_{\mu}=\frac{8 \pi}{3+2 \omega} T^{\mu}{ }_{\mu}
$$

Where equation (10) can be read as 


$$
R^{\mu v}-\frac{1}{2} g^{\mu v} R=-\frac{8 \pi}{\phi} T^{\mu v}-\frac{\omega}{\phi^{2}}\left(\phi^{; \mu} \phi^{; \nu}-\frac{1}{2} g^{\mu v} \phi ;{ }_{\rho} \phi^{; \rho}\right)-\frac{1}{\phi}\left(\phi^{; \mu ; v}-g^{\mu v} \square \square^{2} \phi\right)
$$

The Bianchi identities then require the gravitational field equation to take the form (15), or equivalently

$$
R_{\mu \nu}=-\frac{8 \pi}{\phi}\left[T_{\mu \nu}-\left(\frac{1+\omega}{3+2 \omega}\right) g_{\mu \nu} T_{\lambda}^{\lambda}\right]-\frac{\omega}{\phi^{2}} \phi_{; \mu} \phi_{; \nu}-\frac{1}{\phi} \phi_{; \mu ; \nu}
$$

This theory becomes equivalent to that of Jordan in the special case of an energy momentum tensor with vanishing trace.

In applying the Brans-Dicke theory to cosmology, we again consider the universe to be smeared out into a homogeneous isotropic continuum. The metric then has the Robertson-Walker form

$$
d \tau^{2}=d t^{2}-R^{2}(t)\left\{\frac{d r^{2}}{1-k r^{2}}+r^{2} d \theta^{2}+r^{2} \sin ^{2} \theta d \phi^{2}\right\}
$$

The energy momentum tensor has the perfect fluid form

$$
T_{\mu \nu}=(\rho+p) U_{\mu} U_{v}+P g_{\mu \nu}
$$

gives the time- time component of equation (16) as

$$
\frac{3 \ddot{R}}{R}=-\frac{8 \pi}{(3+2 \omega) \phi}\{(2+\omega) \rho+3(1+\omega) P\}-\frac{\omega \dot{\phi}}{\phi^{2}}-\frac{\ddot{\phi}}{\phi}
$$

while the space-space components of equation (16) gives

$$
-\frac{\ddot{R}}{R}-\frac{2 \dot{R}^{2}}{R^{2}}-\frac{2 k}{R^{2}}=-\frac{8 \pi}{(3+2 \omega) \phi}\{(1+\omega) \rho-\omega P\}+\frac{\dot{\phi} \dot{R}}{\phi R}
$$

And the time-space components simply say that zero equals zero. The field equation (14) for $_{\phi}$ here reads

$$
\frac{d}{d t}\left(\dot{\phi} R^{3}\right)=\frac{4 \pi}{(3+2 \omega)}(\rho-3 P) R^{3}
$$

Now we derive the conservation equation:

We have the solution of time-time component is

$$
\frac{\dot{R}^{2}}{R_{2}}+\frac{k}{R^{2}}=\frac{8 \pi \rho}{3}
$$

Differentiating equation (22) with respect to $t$ we get

$$
\dot{\rho}+3 H(\rho+P)=0
$$


If $\rho=3 P$ then from equation (22) we get,

$$
\frac{\dot{R}^{2}}{R^{2}}+\frac{k}{R^{2}}=\frac{8 \pi \rho}{3 \phi}-\frac{\dot{\phi} \dot{R}}{\phi R}+\frac{\omega \dot{\phi}^{2}}{6 \phi^{2}}
$$

We can recover (19) and (20) from the derivative of (21), so the fundamental equations of the Brans-Dicke cosmology may be taken as (21), (23) and (24), plus an equation of state giving $P$ as a function of $\rho$. In addition, equation $G=\left(\frac{2 \omega+4}{2 \omega+3}\right) \varsigma$ shows that the gravitational "constant" measured by the observation of slowly moving particles or in time dilation experiments is

$$
G=\left(\frac{2 \omega+4}{2 \omega+3}\right) \phi^{-1}
$$

where ${ }_{\varsigma}=\frac{1}{\phi}$; it is called coordinate and it is function of time.

\section{New Result}

We have an idea about the homogeneous and isotropic Brans-Dicke cosmological solutions. Now we show that Mach's assumption leads to power-law solutions in the Euclidean case.

Over the years there has been a great deal of interest in Mach's principle, viz., that the inertia of a body and hence its gravitational properties ought to arise from the rest of the matter in the universe. There are many different formulations of Mach's principle, and one of these is the Whitrow-Randall relation (WRR) [18] given by Berman and Som[2] as

$$
\frac{G M}{R} \sim 1
$$

Where $M$ and $R$ stand for the mass and radius, respectively, of the visible universe. Relation (26) suggests either that ${ }_{M} / R$ is constant, or that the gravitational "constant" $G$ should vary. The later idea found fruition in Brans-Dicke theory (BDT) in which $G$ is related to a scalar field which is determined by the distribution of matter of the universe. Of all the alternatives to general relativity, BDT is considered most seriously.

The field equations in Brans-Dicke theory(BDT) for the Robertson-Walker metric and for a perfect fluid are, in suitable units

$$
\ddot{\phi}+3 \dot{\phi} \frac{\dot{R}}{R}=\frac{4-3 \gamma}{3+2 \omega} \rho
$$

From equation (22) we obtain

$$
\dot{\rho}+3 \frac{\dot{R}}{R}(\rho+P)=0 ; \text { As }_{H}=\frac{\dot{R}}{R}
$$




$$
\left(\frac{\dot{R}}{R}\right)^{2}+\frac{k}{R^{2}}=\frac{\rho}{3 \phi}-\frac{\dot{\phi}}{\phi} \frac{\dot{R}}{R}+\frac{\omega}{6}\left(\frac{\dot{\phi}}{\phi}\right)^{2}
$$

The gravitational parameter $G$ is related to ${ }_{\phi}$ by

$$
G=\frac{2 \omega+4}{2 \omega+3} \frac{1}{\phi} \equiv \frac{A}{\phi}
$$

Where $_{A}=(2 \omega+4) /(2 \omega+3)$. The WRR may also be written in the form

$$
G \rho=6 / t^{2}
$$

The deceleration parameter $q$ is defined by

$$
q=-\frac{R \ddot{R}}{\dot{R}^{2}}
$$

Berman and Som derived their solutions by assuming a constant deceleration parameter as well as the WRR. Here, we consider the Euclidean case $k=0$. We show that by assuming only the WRR, we obtain solution in which deceleration parameter is automatically constant.

From the conservation equation we have

$$
\dot{\rho}+3 \frac{\dot{R}}{R}(\rho+P)=0
$$

For the matter dominant universe $P=0$ then

$$
\begin{aligned}
& \dot{\rho}+3 \frac{\dot{R}}{R}(\rho+0)=0 \\
& \rho \propto R^{-3}
\end{aligned}
$$

Also for radiation dominant universe we have $P=\frac{1}{3} \rho$ then

$$
\begin{aligned}
& \dot{\rho}+3 \frac{\dot{R}}{R}\left(\rho+\frac{1}{3} \rho\right)=0 \\
& \rho \propto R^{-4}
\end{aligned}
$$

which is the solution for the radiation dominant universe

The conservation equation (31a), for generalization of (32) and (33) we write the following relation:

$$
\rho=J R^{-3 \gamma}
$$

where $_{1} \leq \gamma \leq 2$ and $J=$ constant 


$$
\text { Or } \rho=J / R^{3 \gamma}
$$

Now substituting equation (30) and (34) in equation (31) we get

$$
\begin{aligned}
& \frac{A}{\phi} \frac{J}{R^{3 \gamma}}=\frac{6}{t^{2}} \\
& \text { Or, } \phi=\frac{A J}{6} \frac{t^{2}}{R^{3 \gamma}}
\end{aligned}
$$

Again differentiating (35) with respect to $t$ we get

$$
\dot{\phi}=\frac{A J}{3} \frac{t}{R^{3 \gamma}}
$$

Putting $k=0$ in equation (29) we get

$$
\left(\frac{\dot{R}}{R}\right)^{2}+0=\frac{\rho}{3 \phi}-\frac{\dot{\phi}}{\phi} \frac{\dot{R}}{R}+\frac{\omega}{6}\left(\frac{\dot{\phi}}{\phi}\right)^{2}
$$

Now,

$$
\frac{\rho}{3 \phi}=\frac{1}{3} \frac{J / R^{3 \gamma}}{A J t^{2} / 6 R^{3 \gamma}}=\frac{2}{A t^{2}}
$$

Also,

$$
\begin{aligned}
& \frac{\dot{\phi}}{\phi}=\frac{A J t / 3 R^{3 \lambda}}{A J t^{2} / 6 R^{3 \gamma}}=\frac{2}{t} \\
& \therefore\left(\frac{\dot{\phi}}{\phi}\right)^{2}=\frac{4}{t^{2}}
\end{aligned}
$$

By substituting these values in (37) we get

$$
H^{2}+\frac{2}{t} H-\frac{2}{t^{2}}\left(\frac{1}{A}+\frac{\omega}{3}\right)=0
$$

Where $_{H} \equiv \frac{\dot{R}}{R}$ is the Hubble parameter. The solution is

$$
H=\frac{-2 \pm \sqrt{4+8 / A+8 \omega / 3}}{2} \cdot \frac{1}{t}
$$

it has been shown that in order for structure formation to occur in the power-law inflationary scenario, we must have $\omega \geq 8$. Then considering negative sign we get 


$$
\begin{aligned}
& H=\frac{-2-\sqrt{4+8 / A+8 \omega / 3}}{2} \cdot \frac{1}{t} \\
& R=\zeta t^{-\xi}
\end{aligned}
$$

Also considering positive sign we get

$$
R=\zeta t^{\xi}
$$

Where ${ }_{\zeta}=$ constant of integration.

\section{Concluding Remarks:}

We have re-examined the Robertson-Walker cosmological models in Brans- Dicke theory that satisfies Mach's principle in the form of the Whitrow Randall's relation (WRR). The error in the paper of Berman and Som has been corrected and we have extended their results. This enabled us to find the more general solutions in the form of power law by obtaining the equation (40). Such power-law solutions appeared to have been first found by Nariai[9]. As a result the cosmic scale factor grows as a large power of time rather than exponentially (de-sitter model) [4]. This means that in extended inflation the physical volume of space remaining in the false vacuum grows only as power of time and not exponentially and unlike Guth's[1] original model, bubble nucleation can convert all of space to the true vacuum. The extended inflation is not without problems, most notably "the big bubble problem" [6]. Inflation ends through bubble nucleation and percolation; the faster the universe expands, determined by the Brans-Dicke parameter $\omega$, the broader the distribution of bubble sizes and the higher the level of in homogeneity. Achieving sufficient homogeneity requires $\omega$ to be less than about 20; unfortunately, solar-system tests of JBD (Jordan Brans-Dicke) theory constrain $\omega$ to be greater than about 500. But in our problem we simply consider the value of the parameter $\omega \geq 8$ for the power-law expansion.

\section{REFERENCES}

1. A.W. Guth;Phys. Rev. D 23, 347(198).

2. Berman, M.S., and Som, M.M. (1990). Gen.Rel, Grav.22, 625.

3. C. Brans and R. H. Dicke. 1961.'Mach's' principle and a relativistic theory of gravitational phys, Rev. 124,125 .

4. De-sitter, W. Mon. Not. Roy. Astr. Soc.78, 3-28 (1907).

5. D.W Sciama. 1953 'On the origin of inertia' Mon. Not.Roy.Astron.Soc. 3,34

6. E.J. Weienberg, phys, Rev. D 40, 3950(1989).

7. E Mach 1893. The science of Mechanics (Chicago open court).

8. J.V. Narlikar, 2002. Cambridge University. Press.

9. Nariai, H. (1968). Prog. Theor. Phys. 40, 49;(949). Prog. Theor. Phys. 42,544.

10. P.A.M. Dirac. 1937. ‘The Cosmological Constants' Nature 139, 323. 
11. P.A.M. Dirac. 1938. ‘A new Basis of Cosmology’ press. Roy, Soc.AI 65,199.

12. P.A.M. Dirac. 1973. 'Long range forces and broken symmetries' Press. Roy, Soc. A333, 403

13. P.A.M. Dirac. 1974. 'Cosmological models and the large number hypothesis' Press. Roy, Soc. A 338, 439.

14. R.H. Dicke. 1968 "Scalar tensor gravitation and the cosmic free-ball Ap.J.152.1

15. R.H. Dicke. 1962 'Mach's principle and invariance under transformation of unity, phys. Rev.125.2163

16. R.H. Dicke1962. "Implications for cosmology of stellar and galactic evolution rates" Rev. Mod. Phys. 34, 110

17. Steven Weienberg, (1972) Gravitation and Cosmology: Principles and Applications of the General theory of Relativity. John Wiley and Sons, inc. United States of America.

18. Whitrow, G. (1946). Nature 158, 165; Whitrow, G. and Randall. D. (1951). Mon. Not, R. Astr. Soc.111, 455. 